\section{POS0731 CLINICAL ASSOCIATIONS OF COGNITIVE DYSFUNCTION IN SYSTEMIC LUPUS ERYTHEMATOSUS (SLE)}

S. Raghunath ${ }^{1,2}$, V. Golder ${ }^{1,2}$, R. Kandane-Rathnayake ${ }^{1}$, E. F. Morand ${ }^{1,2}$, Y. Glikmann-Johnston ${ }^{3}$, J. C. Stout ${ }^{3}$, A. Hoi ${ }^{1,2} .{ }^{1}$ Monash University, Centre for Inflammatory Diseases, Melbourne, Australia; ${ }^{2}$ Monash Health, Rheumatology Department, Melbourne, Australia; ${ }^{3}$ Monash University, Turner Institute for Brain and Mental Health, School of Psychological Sciences, Melbourne, Australia

Background: Cognitive symptoms are commonly reported by SLE patients, but the prevalence and clinical associations of cognitive dysfunction (CD) is poorly understood.

Objectives: To examine the relationship between SLE disease parameters and CD.

Methods: Patients with SLE were consecutively recruited from 2018-2020. Cognitive assessment was performed using the one-hour conventional neuropsychiatric test battery recommended by the American College of Rheumatology for SLE ${ }^{1}$. Three binary definitions of $C D$ and seven cognitive test z-scores were included in analysis. Clinical parameters included disease activity (SLEDAI-2K) and damage (SDI). Multivariate regression analysis was performed to determine associations with cognitive endpoints using variables with $p<0.1$ on univariate testing; likelihood ratio tests were used to select for inclusion from collinear pairs. A subgroup univariate analysis was performed to look for associations with interferon gene signature (IGS) and metabolic indices.

Results: 91 SLE patients and 48 age, sex, and premorbid IQ matched controls were recruited. The median (range) age was 45 (21-64) and median disease duration 13 years $(0.2-39)$. 60\% were Caucasian and the rest predominantly Asian, and all had good English proficiency. Prevalence of $C D$ varied based on definition used; $18 \%$ had $\geq 2$ cognitive domains $\geq 2$ SD below the healthy contro mean, whereas $48 \%$ were considered impaired if this threshold was reduced to $\geq 1.5 \mathrm{SD}$ below. Age and premorbid IQ were significantly associated with multiple cognitive endpoints, and SDI was associated with four endpoints including the most severe definition of $C D$. Increased parity and past strokes correlated with poorer cognition in two endpoints. In contrast, clinical variables such as SLEDAI, antiphospholipid antibodies and past seizures only correlated with one endpoint each. There were no associations with other antibodies or any medications including glucocorticoids. Subgroup analysis showed univariate associations of $\mathrm{CD}$ with increased waist circumference, hypertriglyceridemia and low IGS. Conclusion: Clinical associations of CD in SLE vary according to the cognitive domain or impairment definition used. SDI was the disease-related factor most consistently associated with $C D$, while IGS was negatively and metabolic features positively associated with CD. Studies of CD in SLE should use standardised methods to determine $C D$.

REFERENCES:

[1] Liang et al. The American College of Rheumatology nomenclature and case definitions for neuropsychiatric lupus syndromes. Arthritis Rheum. 1999;42(4).

Acknowledgements: We would like to acknowledge the patients who contribute to the Australian Lupus Registry and Biobank (ALRB) and participated in this study. The ALRB has received sponsorship from AstraZeneca, UCB and Arthritis Victoria.

Disclosure of Interests: None declared

DOI: 10.1136/annrheumdis-2021-eular.1487

\section{\begin{tabular}{|l|l}
\hline POS0732 IDENTIFICATION OF AUTOPHAGY-RELATED \\
\hline
\end{tabular} PHENOTYPES IN PRIMARY SJOGREN'S SYNDROME}

R. Zhao ${ }^{1,2,3}$, S. X. Zhang ${ }^{1,2,3}$, J. Qiao ${ }^{1,2,3}$, S. Song ${ }^{1,2,3}$, Y. Zhang ${ }^{1,2,3}$, M. J. Chang ${ }^{1,2,3}$, Q. Wang ${ }^{4,5}$, G. Y. Liu', P. F. He ${ }^{5}$, X. Li $^{1,2,3}{ }^{1}{ }^{1}$ The Second Hospital of Shanxi Medical University, Department of Rheumatology, Taiyuan, China; ${ }^{2}$ Shanxi Li Xiaofeng Medical Groups, Department of Rheumatology, Taiyuan, China; ${ }^{3}$ Ministry of Education, Key laboratory of Cellular Physiology at Shanxi Medical University, Taiyuan, China; ${ }^{4}$ Shanxi Medical University, Department of Basic Medical College, Taiyuan, China; ${ }^{5}$ Shanxi Medical University, Medical Data Sciences, Taiyuan, China

Background: Primary Sjogren's syndrome (pSS) is a chronic systemic autoimmune disease characterized by disorders of effector $T$ cell subpopulations such as Th1, Th2, Th17, regulatory T cells, and follicular helper T cells ${ }^{12}$. Autophagy is an evolutionarily conserved self-digestion process that plays an important role in $\mathrm{T}$ cell-mediated immune response ${ }^{3}$. The relationship between autophagy and $\mathrm{T}$ cell subsets was unclear in pSS up till now.

Objectives: To landscape the autophagy-related multiple gene expression signature in pSS classification and discover the influence of autophagy in T cell subsets.

Methods: Gene expression profiles of pSS samples (GSE66795, GSE51092, GSE154926) were acquired from GEO database. A set of significant G-ATGs were intersected from the global gene of patients and 232 autophagy genes (ATGs) which were obtained from the Human Autophagy Database (HADb, http://www.autophagy.lu/). In training dataset (GSE66795, including 155 patients and 29 healthy controls), non-negative matrix factorization was used to divided patients by G-ATGs expression microarray data. An autophagy score model divided patients into the high-autophagy score and low groups by sSGSEA scores of gene according to normalized G-ATGs training data. Further, new classifications were validated by both peripheral blood samples (GSE51092, 90 patients) and salivary gland tissue (GSE154926, 43 participants).

Results: Two distinct subtypes were identified and validated by 206 selected significant G-ATGs in training datasets (figure $1 \mathrm{~A}, \mathrm{~B}$ ) and validation datasets according to the autophagy score (figure 1D,E,F) Combined with clinical information of salivary gland dataset, it was found that most patients with early pSS were grouped in the high autophagy, while advanced patients were grouped in the low (figure 1G). Patients in high-autophagy group had higher levels of Treg cells and Th2 cells but lower concentrations of Th17 and Th1 in peripheral blood (figure $1 \mathrm{C}, \mathrm{P}<0.05$ ). Similar results were also observed in salivary gland tissue (figure $1 \mathrm{H}, \mathrm{P}<0.05$ ).

Conclusion: Patients with different autophagy status differs from each other. Autophagy is closely corelated with lymphocyte subpopulations in patients with pSS. This work may help inform therapeutic decision-making for the treatment of pSS.

Table 1. Multivariate Analysis of Clinical Predictors of Cognitive Dysfunction in SLE

Cognitive Dysfunction Definitions $^{1}$

\begin{tabular}{|c|c|c|c|c|c|c|c|c|c|}
\hline $\begin{array}{c}2 \text { domains } \\
-1.5 \mathrm{SD}\end{array}$ & $\begin{array}{l}1 \text { domain } \\
-2 \mathrm{SD}\end{array}$ & $\begin{array}{c}2 \text { domains } \\
-2 \mathrm{SD}\end{array}$ & $\begin{array}{c}\text { Visual } \\
\text { Memory } \\
\text { (ROCF recall) }\end{array}$ & $\begin{array}{l}\text { Verbal } \\
\text { Memory } \\
\text { (CVLT 1-5) }\end{array}$ & $\begin{array}{c}\text { Verbal } \\
\text { Fluency } \\
\text { (COWAT FAS) }\end{array}$ & $\begin{array}{l}\text { Working } \\
\text { Memory } \\
\text { (LNS score) }\end{array}$ & $\begin{array}{c}\text { Processing } \\
\text { Speed } \\
\text { (Coding score) }\end{array}$ & $\begin{array}{c}\text { Complex } \\
\text { attention } \\
\text { (TMT B time) }\end{array}$ & $\begin{array}{c}\text { Psychomotor } \\
\text { Speed } \\
\text { (Finger tap) }\end{array}$ \\
\hline
\end{tabular}

Odds ratio

Age

Age
Premorbid IQ
SLEDAI

SLEDAI

Time-adjusted mean SLEDAI

PGA

SDI

Past stroke or TIA

Past seizures

Methotrexate

Anti Ro

Anti-cardiolipin

Any APLS abs

Parity

Substance use (logistic regression)

Coefficient (linear regression)

\begin{tabular}{|c|c|c|c|c|c|c|c|c|c|}
\hline $1.06^{*}$ & $1.06^{*}$ & & $-0.03^{*}$ & & & & $-0.014^{*}$ & & $-0.03^{\star \star \star}$ \\
\hline \multirow[t]{4}{*}{$\begin{array}{c}\mathbf{0 . 8 9}^{\star *} \\
0.86\end{array}$} & $\begin{array}{c}0.86^{\star *} \\
0.89\end{array}$ & $0.85^{\star}$ & $0.056^{\text {** }}$ & $0.044^{\star *}$ & $0.072^{\text {** }}$ & $0.028^{\star *}$ & $0.026^{*}$ & $0.05^{\star \star}$ & 0.017 \\
\hline & & & & $0.14^{\star \star}$ & & & & & -0.012 \\
\hline & & & & & & & & $0.79^{\star \star}$ & 0.44 \\
\hline & & $1.91^{*}$ & $-0.25^{\star *}$ & & & & $-0.15^{\star *}$ & $-0.22^{\star \star \star}$ & \\
\hline \multirow[t]{2}{*}{7.94} & $13.19^{*}$ & & & & & $-0.97^{\star \star}$ & & & \\
\hline & & & & $-0.65^{\star}$ & & & & & -0.24 \\
\hline
\end{tabular}

$$
0.22
$$

$0.037^{* *}$

$2.21^{\star *}$

2.58
$0.38^{*}$

$-0.051$

$-0.49^{\star}$

$-0.22^{\star \star}$

$-0.62^{*}$

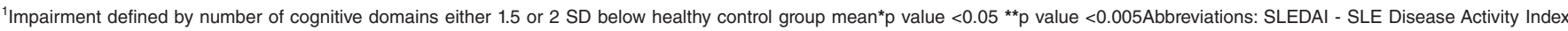
PGA - Physician global assessment, SDI - SLE International Collaborating Clinics Damage Index, TIA - Transient Ischaemic Attack, abs - Antibodies, APLS - Antiphospholipid Syndrome, ROCF - Rey-Ostrrieth Complex Figure Test, CVLT - California Verbal Learning Test, COWAT - Controlled Oral Word Association Test, LNS and Coding - Letter Number Sequencing \& Coding subsets of Wechsler Adult Intelligence Scale IV, TMT - Trail Making Test 